\title{
Plant genetic engineering and biotechnology: a sustainable solution for future food security and industry
}

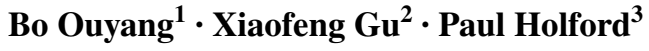

Published online: 20 July 2017

(C) Springer Science+Business Media B.V. 2017

Genetic engineering and biotechnology are becoming widely used in crop improvement and have provided a means by which increased yields of food and fiber can be produced in an environmentally sustainable manner. In addition, these techniques have allowed us to gain great insights into the networks of genes that result in the production of various bioproducts that can be beneficial for human health and environment. The past 5 years have witnessed substantial breakthroughs in plant genetic engineering and biotechnology. In addition to the manipulation of protein coding genes, microRNAs have proved to be promising targets in crop improvement, and the clustered regularly interspaced short palindromic repeat (CRISPR)-associated endonuclease 9 (CRISPR/Cas9) system has emerged like a radiant sunrise, and has greatly facilitated the targeted modification of specific traits. Reverse breeding technologies are also providing a means of accelerating breeding, allowing new cultivars to be produced to combat the

This special issue on Plant Genetic Engineering and Biotechnology is edited by: Bo Ouyang, Xiaofeng Gu, and Paul Holford.

Bo Ouyang

bouy@mail.hzau.edu.cn

Xiaofeng $\mathrm{Gu}$

guxiaofeng@caas.cn

Paul Holford

P.Holford@westernsydney.edu.au

1 College of Horticulture and Forestry Sciences, Huazhong Agricultural University, Wuhan 430070, China

2 Biotechnology Research Institute, The Chinese Academy of Agricultural Sciences, Beijing 100081, China

3 School of Science and Health, Western Sydney University, Penrith, NSW 2751, Australia challenges associated with a changing climate. This special issue focuses on plant genetic improvement, especially on the tolerance of plants to stress.

Three review papers are included in this special issue. Gerszberg and Hnatuszko-Konka (2017) present an excellent review of the genetic engineering of abiotic stress in tomato. Being adversely affected by various abiotic stresses, tomato provides an excellent research model for the study of these stress tolerance in fleshy fruits. Much effort has been made using genetic engineering to understand stress-related gene expression in tomato, and as such, deserves a comprehensive review. Unlike other reviews that divide this topic into different types of stress, this review focuses on the molecular players involved in stress responses. The review briefly introduces the physiological basis of abiotic stress tolerance in plants then details the approaches to genetic engineering and the achievements in understanding and improving stress tolerance. The review includes: (1) the genetic regulation of various metabolites, including mannitol, glycine betaine, glutathione, unsaturated fatty acids, osmotine, polyamines, and trehalose; (2) the regulation of genes associated with hormone pathways (e.g. ethylene), water channels (e.g. aquaporin) and ion transport; (3) the manipulation of heat shock proteins; (4) the regulation of enzymes in antioxidant systems, such as oxidoreductase, catalase, ascorbate peroxidase, and superoxide dismutase; and (5) the genetic transformation of regulatory genes (e.g. transcription factors and kinases) and other components of signaling systems (e.g. systemin, expansin, and late embryogenesis abundant protein).This review is likely to serve as an informative reference for abiotic stress research in tomato.

Singh et al. (2016) present a short review of the roles of cross-talk between microRNA and nitric oxide (NO) in signaling associated with stress responses. MicroRNAs are 
emerging as an essential player in plant stress responses, while NO signaling has been identified as a new player in these processes. To help readers to overview of the contents, the authors present three models in the review that illustrate the signaling pathways involved in drought/coldinduced, NO-miRNA mediated gene expression and the dual but opposite regulation of miR398 under oxidative stress or copper deprivation.

Savadi et al. (2016) summarize the genetic approaches used in the improvement of plant oil content. Vegetable oils are important for human diets and as a raw material in industrial applications and, more recently, have become significant as biofuels. Hundreds of genes are involved in the enzymatic and regulatory pathways controlling lipid metabolism; therefore, the genetic manipulation of oil content is a complex process. Nevertheless, various approaches have been applied to enhance oil content, including manipulation of triacylglycerol/fatty acids (TAG/FA) synthesis, modulation of carbon flux towards TAG/FA biosynthesis, the alteration of transcription factors, the manipulation of oil bodies, the extension of the duration of oil biosynthesis, the introduction of a novel TAG synthesis pathway, the reduction of breaking down the stored lipids, increasing sink size for oil accumulation, and pyramiding multiple genes in oil content regulation. The information is clearly integrated in to a few figures and the genes are listed in detail by a table.

This special issue also includes two papers demonstrating different strategies of exploring functional genes for plant genetic engineering research. Today, two major strategies, reverse genetic and forward genetic approaches, are intensively applied to identify genes, and these two strategies are complimentary to each other. RNA profiling using microarray or high-throughput sequencing is widely used to identify genes involved in a particular biological process, such as protein-coding genes or non-coding RNAs that respond to a particular stress. This strategy is classified into reverse genetics. As a case of this approach, Xie et al. (2016) have profiled microRNA expression by highthroughput sequencing of Masson pine (Pinus massoniana) inoculated with the pine wilt pathogen, Bursaphelenchus xylophilus. They identified ten differentially expressed microRNAs due to pathogen attack that are good candidates for further functional analysis. MicroRNAs have been found to be intensively involved in plant growth and development as well as stress responses, and their identification is a first step to their utilization. Map-based cloning is a typical forward genetic approach to identifying agronomically important genes. Using this technique, Jiao et al. (2017) used an $F_{2}$ population of cucumber to genetically map the green-skin trait. The skin color of cucumber is an important organoleptic quality that affects customer preference. Jiao et al. (2017) utilized various biochemical and molecular techniques to functionally analyze the strong candidate gene, APRR2. Collectively, their data suggest that APRR2 together with two Class-I KNOTTED1-like homeobox (KNOX) genes (TKN4, TKN2) contribute to the mechanisms related to chloroplast development in cucumber skin. The isolation and characterization of APRR2 provides knowledge of a good candidate gene for the genetic modification of organoleptic quality in cucumber.

Beside the identification of functional genes in genomes, the establishment of an efficient regeneration system for a target species is also a prerequisite for crop improvement via genetic transformation. Although highly efficient regeneration and transformation systems have been developed for quite a number of crops, many plant species remain to be recalcitrant to regeneration and transformation, including some economically important specie (i.e. Capsicum and citrus). Here, in this special issue, Bao et al. (2017) have developed a rapid and efficient in vitro shoot regeneration protocol for London plane tree (Platanus acerifolia Willd.) using cotyledons as explants. After much work on the optimization of plant growth regulator recombinations and seedling age, they established a practical protocol for shoot regeneration of this recalcitrant species. This system will hopefully accelerate the genetic transformation of $P$. acerifolia.

Finally, this special issue contains two papers using transgenic plants to study stress tolerance genes. Fu et al. (2017) generated transgenic Arabidopsis plants that overexpressed arginine decarboxylase (ADC), a rate-limiting enzyme in putrescine (Put) synthesis thereby resulting in improved tolerance to salt stress. Under salt stress, these plants performed better than the untransformed control plants and ADC deficient mutants, had higher chlorophyll contents, and lower levels of malonaldehyde, superoxide and hydrogen peroxide. Superoxide dismutase (SOD) and catalase (CAT) levels were also significantly higher in the transgenic lines. These results strongly suggest that salt stress induces the expression of AtADC, thus increasing Put accumulation; consequently, Put regulates the activities of SOD and CAT to eliminate reactive oxygen species and enhance salt tolerance. In the other study, $\mathrm{Yu}$ et al. (2017) investigated the function of betaine aldehyde dehydrogenase (BADH) from a xerophytic, leguminous shrub endemic to the Mid-Asia deserts, Ammopiptanthus nanus, that is tolerant to high salinity, extreme dryness and temperatures. Heterologous expression of $A n B A D H$ in an Arabidopsis mutant deficient in aldehyde dehydrogenase significantly enhanced tolerance to salt and drought stress. Under the stress conditions, the transgenic lines exhibited a more robust root system and a larger fresh weight, higher glycine betaine, proline and relative water contents, and lower relative electrolyte leakage and malondialdehyde contents. All these results demonstrate that the AnBADH 
encodes a functional BADH, a gene that appears to play an important role in the adaptation of A. nanus to harsh environments.

The range of molecular breeding methodologies and technologies featured in this special issue, will continue to expand allowing genetic engineering and biotechnology to play an increasing role in plant improvement as well as finding further applications in industry, medicine and environmental protection. Plant-based bioreactors and metabolic engineering will help to deliver beneficial medical products. Genetic engineering and biotechnology will open new avenues to the production of bioproducts, such as biofuels and bioplastics, with reduced inputs and costs of production and resulting environmental benefits. We hope that this special issue, focusing on plant genetic engineering and biotechnology, will stimulate more research in these areas, and we would like to thank the authors and reviewers for their contributions and to Plant Growth Regulation for the opportunity to present this special issue on this continuously evolving topic.

\section{References}

Bao Z, Zhang Y, Shao C, Zhang J, Liu G, Bao M (2017) A rapid and efficient in vitro shoot regeneration protocol using cotyledons of
London plane tree (Platanus acerifolia Willd.). Plant Growth Regul. doi:10.1007/s10725-017-0303-2

Fu Y, Guo C, Wu H, Chen C (2017) Arginine decarboxylase ADC2 enhances salt tolerance through increasing ROS scavenging enzyme activity in Arabidopsis thaliana. Plant Growth Regul. doi:10.1007/s10725-017-0293-0

Gerszberg A, Hnatuszko-Konka K (2017) Tomato tolerance to abiotic stress: a review of most often engineered target sequences. Plant Growth Regul. doi:10.1007/s10725-017-0251-x

Jiao J, Liu H, Liu J, Cui M, Xu J, Meng H, Li Y, Chen S, Cheng Z (2017) Identification and functional characterization of APRR2 controlling green immature fruit color in cucumber (Cucumis sativus L.). Plant Growth Regul. doi:10.1007/s10725-017-0304-1

Savadi S, Lambani N, Kashyap PL, Bisht DS (2016) Genetic engineering approaches to enhance oil content in oilseed crops. Plant Growth Regul. doi:10.1007/s10725-016-0236-1

Singh R, Parihar P, Singh S, Singh MPVVB, Singh VP, Prasad SM (2016) Micro RNAs and nitric oxide cross talk in stress tolerance in plants. Plant Growth Regul. doi:10.1007/s10725-016-0190-y

Xie W, Huang A, Li H, Feng L, Zhang F, Guo W (2016) Identification and comparative analysis of microRNAs in Pinus massoniana infected by Bursaphelenchus xylophilus. Plant Growth Regul. doi:10.1007/s10725-016-0221-8

Yu HQ, Zhou XY, Wang YG, Zhou SF, Fu FL, Li WC (2017) A betaine aldehyde dehydrogenase gene from Ammopiptanthus nanus enhances tolerance of Arabidopsis to high salt and drought stresses. Plant Growth Regul. doi:10.1007/s10725-016-0245-0 